\title{
Packaging Design in Conservation-Minded Society
}

\author{
Chengrui Duan, Hongliang Xing and Xiaodan Li \\ College of Design \& Art of Shenyang Aerospace University
}

Keywords: Economical; Packaging design; Electronic products.

\begin{abstract}
Along with decrease and shortage of earth resources, economical packages enter into people's life which will lead fashion in the future life and change consumption habits of consumers. It is imperative to simplify some unnecessarily luxurious packages and it is inevitable to make economical designs and de-packaging treatments to packages of different products like foods and electronic products in the future.
\end{abstract}

\section{Introduction}

In recent years, along with descending global economic development speed, all industries are under influences of different levels. Without exception, packaging industry is pushed aside by upstream and downstream industries during economic adjustments, which again gives rise to attentions of manufacturers and consumers on economical packaging. Thus economical packages, accompanied with fashion, come into people's life in recent years.

\section{Over Packaging and Consumption}

In the middle of last century, China was economically backward. At that time, our country was at the initial stage of liberation which the economy just started to grow and was difficult, so there were no strict standard requirements for products packaging and the only requirement was to sufficiently ensure safety and transportation of products, let alone specific requirements on three major elements of figure, character and color. Along with social transformation and market economy reform of China, visual culture also took the lead in joining in market competitions as well, and represented cultural characteristics of certain consumer society [1]. Package design entered into a developing stage and improvements on colors and packaging materials were made. After the reform and opening-up in 1980s, rapid growth of economic standard led to big improvements of people's life. A small number of people obtained leaps in economy in face of waves of reform, thus possessing certain strengths in consumption. Following this kind of change, consumption concepts of some people were changed from previous simple and straitened to disposable even luxurious or abnormal. Pursuit of luxurious products brought about over packaging[2] in packaging market, which package value and commodity value were imbalanced. Specific manufacturers and designers applied all kinds of expensive materials to packaging instead of simple but functional packages. Blindly pursuing luxurious and exaggerated packages without consideration of price of package itself or waste of scarce resources is still a phenomenon in current market.

Over packaging in current market is mostly in gift packages. Mooncake, as a kind of festival food to be gifted to relatives and friends, its packages turned to luxurious packages in the end of 1990s, multiplying its prices to few tens even several thousands Yuan. A box of a few pieces of mooncakes 
with excessively high price let many people could not afford it. However, these mooncakes had their own market that most of them were given as gifts bought with public funds, which as a consequence encouraged briberies, thus mooncake packages were controlled under strict regulation and control of various policies. In fact, consumers knew that mooncakes in the package did not worth hundreds or thousand Yuan, but due to influence of "saving face" of consumers, they would buy mooncakes with more luxurious packages, thus leading to competitions on luxurious packages of manufacturers. Moreover, costs of over packages are still borne by consumers, this not only damages benefits of consumers, but also is overdrafting social purchasing power. The author can not help thinking of a story called "keeping the glittering casket while giving back the pearls to the seller". According to External Treatise Collections Zuoshang Volume of Han Feizi, "Once one man of Chu sold pearls in Zheng, and his pearls were kept in a beautiful box made from lily magnolia, smoked with laurel, and decorated with pearls, jades, roses and feathers. One man of Zheng bought the box while giving back the pearls to the seller. This can be said that seller is good at sells ing casket while not pearls." [3] Stories of this kind still happen today, and the reason is that extremely beautiful packaging boxes let people ignore the inside boxes. This also reminds the author that in the last century, each family would use some firm and delicate packaging boxes to store all kinds of toys of the kids and sundries. It was in the days with shortage of supplies. But today, as the economy grows rapidly, these delicate boxes become burdens of people's life. Observing those abandoned boxes, a kind of feeling of "tasteless but wasteful to throw it away" arose spontaneously. Over packaging results in wasting of resources and costs abundant human and material resources, and in the end are disposed as domestic rubbishes. The more complicated packaging process is, the more obvious influences to environment it has. Therefore such packages should be improved, and of course, this is related to social concept and consumption habits of the people.

\section{Influences of Over Packaging to Environment}

Aimed at packaging and packaging wastes, European Union issued Directive on Packaging and Packaging Waste and its amendment in 1994. According to Julian Carol, Executive Director of European Packaging and Environmental Agency, the Directive allowed its member countries to take economic measures such as ecological tax and waste disposal tax to reduce product packaging and to promote usage of eco-packaging, but it should not interfere with operation of European internal market. The Directive sets phased objectives for realizing recycling rate of packages: it required the member countries to measure packaging wastes by weight and by the end of 2008, recycling and incineration rate shall reached to at least 55\%. Meanwhile, recycling rates of different packaging materials are different, for example, recycling rates of glass, paper, metal materials, plastic products and wood products are $60 \%, 50 \%, 22.5 \%$ and $15 \%$ respectively[4].

In China, with regard to implementation of Notice of The State Council on Key Task of Building A Conservation-Oriented Society in 2005, China Packaging Federation pointed out that packaging industry belonged to resource consumption industry, $70 \%$ of packaging products were disposable and life cycle of packaging products was shorter than other industrial products. Packaging products would become wastes immediately after use and they cost large resource consumptions. In 2004, gross output of packaging industry in our country was more than 320 billion Yuan, wherein products of about 230 billion Yuan would become packaging wastes. Due to low recycling rate of packaging wastes in our country, many packaging wastes which could have been recycled are not used effectively. According to investigation, packaging wastes in large and medium-sized cities account 
for $1 / 3$ in total volume of city household wastes, which causes serious damages to the environment. Landfills of these wastes occupy plenty of lands, and "white pollution" caused by expended plastics and plastic bags leads to severe influences to the environment of cities, rivers and two sides of railways, which becomes a social disaster[5].

\section{Simple Packaging Under Modern Economy}

Along with global economic crisis, designing is changing quietly. Recently, hamburger packages of KFC, a famous international fast food chain, are changing slightly from original paper boxes to butter papers for food use. This reflects merchants are looking for some effective ways to increase income and reduce expenditures in the situation of declining economic development. In fact, packages of hamburgers and other fast foods generally have only one-minute use, then will lose the packaging functions and turn into wastes. One inevitable aspect of packaging design thus is touched upon, namely influences of packages to environment. Post-treatment and influences to environmental of these fast food packages are more importance than their one-minute life. Recovery processing of paper pulps shall be conducted to waste hamburger packaging boxes. However, thin plastic films attached to surfaces of the boxes increase difficulties in recycling and may lead to environmental pollutions. Applications of butter paper can not only save costs, but also reduce wastes post-treatment work. Evolving from cartons with exquisite prints to simple butter paper packages is no doubt a progressive idea in packaging industry, and shows economical and environmental consciousness and social responsibilities of manufacturers. The Independent in UK, based on statistics, once pointed out that "after the annual shopping for Christmas, a familiar sight appears again in front doors and backyards of London citizens and stores: pine tree branches scattering in disorder, and wastebaskets, packaging boxes and empty wine bottles piling up in heaps. Within 10 days after Christmas, sanitation workers in England would collect about 3 million tons of pine tree branches, packaging boxes and cards, and 0.7 billion glass bottles, 0.5 billion beverage cans and several tens of thousands of food garbage". The Newspaper initiated a movement to appeal British people resist over packaging. The newspaper warned that if over packaging went on, landfills of England would be packed in two years, and what's worse, environmental disruption would aggravate rapidly[6]. Over packaging makes unnecessarily luxurious packages into a kind of gifts and even fashion, thus it is a social responsibility to resist over packaging.

\section{Changes of Packaging of Digital Products}

Along with rapid development of economy in our country, wastes generated from product packages are increasing at a high speed, threatening environmental management and long-term development of our company. In the industry of electronic products, after come out of Sumsang Galaxy tablet phones, simple packages of Samsung mobile phones are highly regarded by global market and packaging and designing industry. Packages of Samsung mobile phones evolved from the original clamshell-type and drawer-type packaging boxes to present up and bottom packaging boxes. Package showed in Fig. 1 possesses features of compact inner structure and small and exquisite appearance, which maximize the use of spaces, thus saving use of raw materials and saving transport and storage space as well. In appearance design, original color design was changed to printing simple and recognizable brand logo on purely black or white groundings. This kind of design is not only convenient for consumers to distinguish models, but also caters to modern stylish of tablet phones 
and delivers product semantics, thus people can experience design style of the inner products through appearance of packaging design.
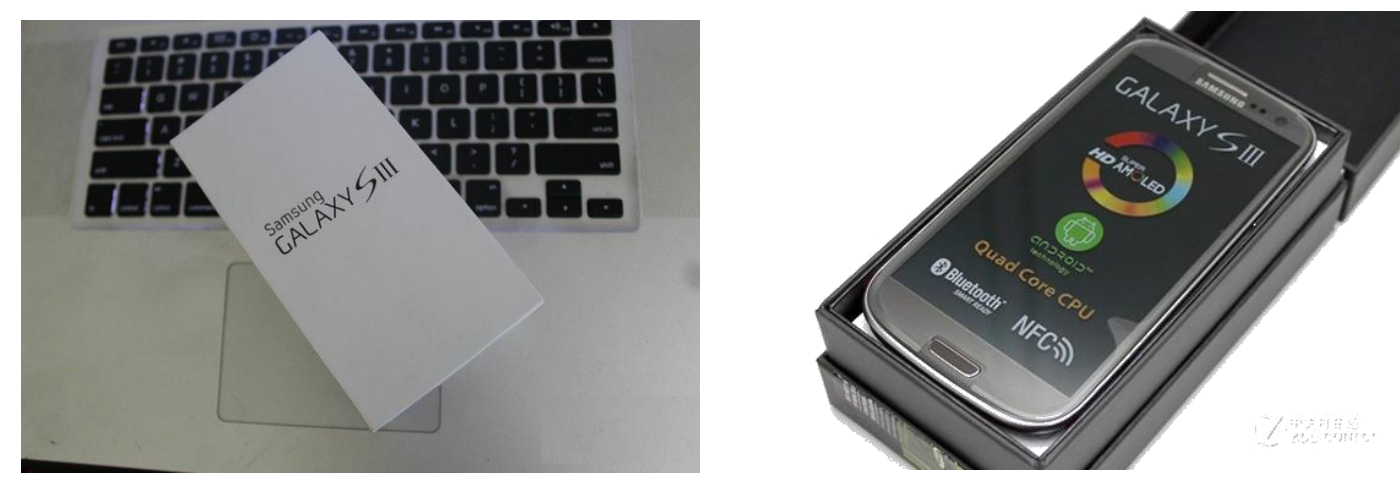

Fig. 1

Electronic products, including mobile phones, are experience-oriented products, so when purchasing such products, consumers shall experience these products first and then determine whether to purchase, while purchase only based on packaging appearance shall be avoided. Therefore, packages of these products are only required to be simple and in good taste and can protect and distinguish products. Besides, text descriptions can also provide striking key points or supplementary instructions to figures, and can make up deficiencies of images[7]. This has been represented in other Samsung products. For instance, in transport package design of Samsung SHS6020 mobile phone, all packaging boxes are uniform model design. This design till takes the corrugated boards as materials, but gets rid of the original printing process of printing colors on the entire board, while printing product models of different groundings with eco-friendly soy ink on papers with unified size pasted on fixed and obvious places instead. Thus products can be distinguished an d protected, and packaging storage and transportation costs of manufacturers are reduced as well.

This kind of packages are accepted by consumers and applied by other manufacturers as well. This represents a good phenomenon that manufacturers leading consumption habits through designing. This is an initiative fashion design which not only helps manufacturers reduce costs but also wins acceptance of consumers and the society. Thus it is not hard to make out influences of big brands to designs and consumption concepts of all countries in the world, so manufacturers with big brands shall shoulder their own social responsibilities to save social resources, lead fashion and bring themselves sound economic benefits.

According to statistics, $9 \%$ of consumers buying digital products will directly take out the products and throw away the packages, 57\% the consumers will throw away the packages after expiry of term of changing or refunding, and $31 \%$ of the consumers will leave the packaging boxes of mobile phones unused at home[8]. It brings a kind or new thinking that whether to simplify sales of product packages, namely, separating products into products with individual packages and products with centralized packages. Consumers needing no packages can choose products with centralized packages, and certain costs will be reduced for them. When buying a assembled computer, what consumers take away is a packaging box of the computer case, while packages accessories like internal CPU, main board, hard disk, video card and memory are thrown away before consumers buy them. Even if with exquisite and complicated processes, what is the purpose of these packages on earth? What if simplify their decorations and just keep the basic functions? Difference between prices of CPUs of assembled computers with bulk packages and individual packages is up to $30 \%$. 
This situation has been existing in sales market of computer accessories for several years, despite problems of purchasing channels, whether this kind of specific consumption pattern will enlighten designing and implementation of economical packages?

\section{Packages Under Influences of Consumption Habits}

In the aspect of European luxuries packages, though prices of famous fashion brands like LV and GUCCI are high, ratio of prices of packaged products to products net products is low. These brands only have a simple brown flannelette packaging bag matched with a paper bag printed with simple logo. Such packages are also popular among consumers in China, so why are costs of packages in China still increasing and staying at a high level without any changes? Direct reason is that manufacturers worry that changing packages will not be accepted by consumers, and it also indirectly related to old consumption habits of consumers. Attracted by high profits, manufacturers forget their social responsibilities and they are not willing to simplify package designing.

Of course these packages are mostly packages of traditional commodities, and influenced by international brands, packages of modern commodities are changed in step with the times. It is a hope that this kind of changes can be made more rapidly. Psychological needs of consumers can be formed through leading and training, and can be weakened or changed by external disturbances[9]. Designers and manufacturers should understand how to lead consumption through leading fashion with their excellent products and designs, while blindly chasing luxurious fashion design will obstruct their steps forward.

\section{Conclusions}

Following economic growth and population changes in our country, economical packaging is imperative. In the meantime of leading fashions, manufacturers and designers shall actively shoulder their social responsibilities to change people's unhealthy consumption habits and help people develop acceptance and preference of economical packages. Designing strategies shall be made based on idea of reducing damages to environment to the greatest extent, thus improving economic, cultural and social development through economical packaging designs[10]. Along with decrease of resources and increase of populations in the future, packaging will inevitably move towards resource-saving, which of course requires supports and coordination of all walks of life in the society.

\section{References:}

[1] Zhou Xian, Visual Culture and Social Transformation [J]; Hundred Schools in Art, No. 5, 2012

[2] Sun Chao, Zhang Ye, Thoughts on Enterprise Social Responsibilities on Over Packaging [J]; Economic Research Guide, No. 26, 2009

[3] Yu Yalin, Enlightenment of "Buying Caskets while giving back pearls"on Positioning of Product Style [J]; Hundred Schools in Arts, No. S1, 2009

[4] Outstanding Achievements of Recycling of Waste Packages in EU [EB/OL]http://news.xinhuanet.com/life/2007-02/09/content_5717188.htm 
[5] Notice of China Packaging Federation on implementation of Notice of The State Council on Key Task of Building A Conservation-Oriented Society [J], Plastic Packaging, No. 06, 2005

[6] Ma Jianguo, Appeal to Resist Over Packaging in UK Newspapers [EB/OL].http://news.xinhuanet.com/life/2007-02/05/

[7] Dong Chuanchao, About Transmission Mechanism of Figures and Words in Visial Communication Designing [J], Hundred Schools of Arts, No. 5, 2012

[8] Jiang Xiaopu, New Thoughts on Packaging Design in Conservation Minded Society [J], Packaging Engineering, No. 12, 2008

[9] Luo Guanglin, Yun Changqing, Packaging and Sale Psychology [M]. Beijing Printing Industry Press, No. 8, 2005

[10] Yang Guoxin, Yang Ping, Explore and Analysis on Green Packaging [J]; Packaging Engineering, No. 6, 2006 